\title{
GRAU DE DEPENDÊNCIA DE CUIDADO: PACIENTES INTERNADOS EM HOSPITAL DE ALTA COMPLEXIDADE
}

\author{
Degree of dependence in care: patients admitted in a hospital with high complexity \\ Grado de dependencia del cuidado: pacientes internados en un hospital de alta \\ complejidad
}

Raquel Gvozd ${ }^{1}$

William Tiago de Oliveira²

Sabine Jenal ${ }^{3}$

Marli Terezinha Oliveira Vannuchi ${ }^{4}$

Maria do Carmo Lourenço Haddad 5

Fernanda Cristina Fortes ${ }^{6}$

\section{RESUMO}

Estudo descritivo transversal com abordagem quantitativa, que objetivou categorizar os pacientes atendidos em unidade de internação de um hospital terciário, quanto à complexidade assistencial e o grau de dependência. 0 instrumento utilizado contempla doze áreas do cuidado, e classifica os pacientes nas seguintes categorias: cuidado intensivo, semi-intensivo, alta dependência, intermediário e mínimo. Observou-se que $68 \%$ da amostra eram do sexo masculino e $32 \%$, do feminino, com mediana de idade de 51,97 anos; $34,9 \%$ dos pacientes estavam restritos ao leito e 32,8\% apresentavam limitação de movimentos; 44,8\% apresentaram presença de solução de continuidade na pele envolvendo tecido subcutâneo e músculo, 29,9\% necessitavam cuidados de alta dependência. 0 estudo mostrou a insuficiência de leitos de UTI, com necessidade da reestruturação da rede de saúde. Pacientes necessitando de cuidados intensivos estão internados em unidades não especializadas, provocando sobrecarga de trabalho para a equipe de saúde e impossibilitando uma assistência de qualidade.

Palavras-chave: Cuidados de enfermagem. Cuidados intensivos. Avaliação em enfermagem. Pacientes internados. Classificação.

\section{Abstract}

A descriptive cross-sectional study with a quantitative approach, which aimed to categorize the patients treated in the admission unit at a tertiary hospital, regarding the care complexity and the degree of dependence. The instrument used covers twelve areas of care, and classifies patients into the following categories: intensive care, semi-intensive, high dependency, intermediate and minimum. It was observed that $68 \%$ were male and $32 \%$ female, mid age of 51.97 years old, $34.9 \%$ of patients were confined to bed and $32.8 \%$ had limitation of movement, $44,8 \%$ showed the presence of continuous flow on the skin involving subcutaneous tissue and muscle, $29.9 \%$ required high dependency care. The study showed a lack of ICU (Intensive Care Unit) beds, the necessity for restructuring the health network. Patients requiring intensive care are admitted at non-specialized units, causing extra work for the health team and causing a low quality care.

\section{Resumen}

Estudio descriptivo trasversal con abordaje cuantitativo, que objetivó categorizar los pacientes atendidos en una unidad de internación de un hospital terciario, en cuanto a la complejidad asistencial y el grado de dependencia. El instrumento utilizado contempla doce áreas del cuidado y clasifica los pacientes en las siguientes categorías: cuidado intensivo; semi-intensivo; alta dependencia; intermediario; mínimo. Se observó que el $68 \%$ de la muestra eran del sexo masculino y el $32 \%$, femenino, con edad media de 51,97 años; el $34,9 \%$ de los pacientes estaban confinados en la cama y el $32,8 \%$ presentaba limitación de movimientos; el 44,8\% tenían presencia de solución de continuidad en la piel envolviendo tejido subcutáneo y músculo y el $29,9 \%$ necesitaba cuidados de alta dependencia. El estudio mostró la insuficiencia de camas de UCI (Unidad de Cuidados Intensivos), con necesidad de reestructuración de la red de salud. Pacientes necesitando cuidados intensivos están internados en unidades no especializadas, provocando sobrecarga de trabajo para el equipo de salud y imposibilitando una asistencia de calidad.
Key words: Nursing Care. Intensive Care. Nursing Assessment. Inpatients . Classification.
Palabras clave: Atención de Enfermería. Cuidados intensivos. Evaluación en Enfermería. Pacientes internos. Clasificación.

\footnotetext{
${ }^{1}$ Enfermeira. Egressa da Residência em Gerência dos Serviços de Enfermagem da Universidade Estadual de Londrina /Hospital Universitário Regional do Norte do Paraná. Aluna do mestrado em Enfermagem da Universidade Estadual de Londrina-PR. Brasil. E-mail: raquelgvozd@yahoo.com.br; Enfermeiro. Egresso da Residência de Gerência dos Serviços de Enfermagem da Universidade Estadual de Londrina. Aluno do Mestrado em Enfermagem da Universidade Estadual de Maringá. Maringá - PR. Brasil. E-mail: oliveirawt@hotmail.com; ${ }^{3}$ Enfermeira. Gerente Multiprofissional da Irmandade Santa Casa de Londrina. Aluna do Doutorado em Enfermagem Fundamental da Escola de Enfermagem de Ribeirão Preto/Universidade de São Paulo. Londrina - PR. Brasil. E-mail: Iorena.jenal@iscal.com.br; ${ }^{4}$ Enfermeira. Professora Doutora do Departamento de Enfermagem da Universidade Estadual de Londrina. Londrina - PR. Brasil. E-mail: haddad@sercomtel.com.br. ;'Enfermeira. Professora Doutora do Departamento de Enfermagem da Universidade Estadual de Londrina. Londrina - PR. Brasil. E-mail: vannuchi@sercomtel.com.br; ${ }^{6}$ Enfermeira. Pós-Graduada em Gerência Assistencial de Enfermagem na Saúde do Adulto. Londrina - PR. Brasil.E-mail: fernanda.fortes@iscal.com.br.
} 


\section{INTRODUÇÃO}

A enfermagem tem sentido cada vez mais a necessidade de utilizar o método científico como base para a organização da sua assistência. Assim, a caracterização dos clientes atendidos quanto às condições clínicas definidoras do grau de dependência de cuidados de enfermagem faz-se necessária, uma vez que embasa a quantificação de provimento de profissionais de enfermagem necessários para uma assistência de qualidade, prevenindo a sobrecarga dos serviços de enfermagem e preservando a saúde do trabalhador ${ }^{1}$.

O Sistema de Classificação de Pacientes (SCP) objetiva agrupar os clientes por complexidade assistencial e surgiu da necessidade das organizações de saúde racionalizar o trabalho e, consequentemente, os recursos humanos e materiais ${ }^{2}$. Esta classificação constitui-se em uma ferramenta gerencial para o planejamento da assistência de enfermagem, auxiliando no envolvimento da equipe com o cuidado prestado, além de possibilitar o controle de materiais e equipamentos utilizados na unidade. Também se constitui em subsídios para a resolução de problemas que os enfermeiros muitas vezes se deparam, tais como a dificuldade em justificar a necessidade de reorganização e contratação de recursos humanos, podendo comprometer a qualidade da assistência prestada ${ }^{3}$.

Os SCP pautados em medidas de diferentes variáveis têm sido implementados na prática dos serviços de enfermagem, contribuindo tanto para a assistência quanto para 0 gerenciamento das unidades hospitalares ${ }^{4}$. Eles se diferenciam na forma de avaliação e no número de categorias avaliadas, mas todos são baseados no cuidado progressivo ao paciente ${ }^{5}$.

0 planejamento adequado da assistência ao paciente ocorre quando se faz uma boa avaliação levando em consideração o grau de dependência. Os modelos assistenciais devem identificar as necessidades dos pacientes, os quais, a depender da complexidade e grau de dependência em relação ao cuidado de enfermagem, exigirão recursos humanos em termos quantitativos e qualitativos adequados ${ }^{6,7}$.

Não obstante, uma das maiores dificuldades encontradas no dia-a-dia de trabalho da equipe de enfermagem nas instituições hospitalares refere-se à insuficiência de profissionais em relação à assistência necessária e a demanda de atendimento cada vez mais complexa dos pacientes ${ }^{8}$.

Existem ainda situações adversas como faltas não previstas e sobrecarga de trabalho, justamente em razão da instabilidade do quadro clínico dos pacientes, que dificultam o trabalho da enfermagem, gerando baixa produtividade, qualidade de assistência prejudicada e alterações na saúde dos trabalhadores?.

Destarte, a complexidade assistencial e o grau de dependência de cuidado de enfermagem têm sido utilizados pelos gerentes de enfermagem como instrumentos que possibilitam adequar o número de profissionais às reais necessidades de assistência aos pacientes, considerando a provisão de recursos humanos, a otimização de custos e, principalmente, a manutenção da qualidade na prestação de serviços e a saúde do trabalhador.

Justifica-se, portanto, a importância da realização desse trabalho, tendo em vista a necessidade da realização do dimensionamento do pessoal de enfermagem adequada ao perfil do paciente atendido, a fim de possibilitar uma assistência segura e de qualidade.

Com base nas considerações anteriores, este estudo teve por objetivo categorizar os pacientes atendidos em uma unidade de internação de um hospital terciário, quanto à complexidade assistencial e o grau de dependência da enfermagem, a fim de se obter dados para elaborar 0 dimensionamento de pessoal.

\section{MÉTODO}

Trata-se de um estudo descritivo transversal realizado em uma unidade de internação de hospital filantrópico terciário localizado no norte do estado do Paraná.

A unidade em estudo possui 38 leitos distribuídos em 15 enfermarias de três leitos, destinada ao atendimento de pacientes adultos de ambos os sexos conveniados ao Sistema Único de Saúde, que apresentam alterações clínicas e cirúrgicas nas diversas especialidades médicas, bem como acometidos por moléstias infecciosas e aqueles considerados como contaminados. Algumas vezes, nesta unidade são internados clientes em estado grave devido à falta de leitos nas Unidades de Terapia Intensivas (UTI).

Os dados para traçar o perfil dos pacientes foram coletados no relatório de estatística hospitalar, por meio do qual se verificou que durante o mês de outubro de 2010 estiveram internados nesta unidade 81 pacientes, sendo que seis deles permaneceram por mais de 100 dias no setor.

0 instrumento utilizado para classificação dos pacientes foi proposto por Fugulin et al. (2005) adaptado por Santos $(2007)^{10}$. Este contempla as seguintes áreas do cuidado: estado mental, oxigenação, sinais vitais, mobilidade, deambulação, alimentação, cuidado corporal, eliminação, terapêutica, integridade cutâneo mucosa/ comprometimento tecidual, uso de curativo e tempo utilizado na sua realização. Desta forma, a graduação da complexidade assistencial é realizada de modo que cada área do cuidado seja pontuada de um a quatro, sendo que a somatória das 12 categorias do cuidado é classificada em: cuidado intensivo (acima de 34 pontos), semi-intensivo (29-34), alta dependência (23-28), intermediário (18-22) e mínimo (1217).

Os dados foram coletados a partir do preenchimento do instrumento aplicado a todos os pacientes internados na referida unidade durante o mês de outubro de 2010. Estes 
pacientes foram avaliados diariamente, no período da manhã, por uma das autoras desse estudo. Foram avaliados em média 31 pacientes por dia, perfazendo um total de 926 avaliações. Ressalta-se que, por se tratar de uma unidade de internação de alta complexidade e longa permanência, o mesmo paciente foi avaliado mais de uma vez neste período.

O estudo foi aprovado pelo Comitê de Ética em Pesquisa da Instituição na qual está alocada a unidade, com parecer favorável e registro no Sistema Nacional de Informação sobre Ética em Pesquisa, CAAE nº 0026.0.083.083-10.

Os dados foram processados através de tabulação no programa Microsoft Office Excel2007 e analisados por porcentagem simples.

\section{RESULTADOS}

Durante o período da coleta de dados, a taxa de ocupação dos leitos foi de $79,5 \%$, não apresentando $100 \%$ pelo fato de a unidade admitir pacientes com moléstias infecciosas, o que impossibilita a internação de pacientes com diferentes bactérias na mesma enfermaria, sendo necessário o isolamento.

A média de permanência nesta unidade durante 0 período da coleta de dados foi de 5,01 dias, excluindo os seis pacientes com mais de 100 dias de internação.

Quanto ao perfil dos pacientes internados, observou-se que $68 \%$ eram do sexo masculino e $32 \%$, do feminino. A mediana de idade desta população permaneceu em 51,97 variando de 14 a 96 anos.

Figura 1 Distribuição das especialidades médicas que internam com maior frequência na unidade em estudo ( $\mathrm{n=81}$ ), Londrina-PR, 2010.

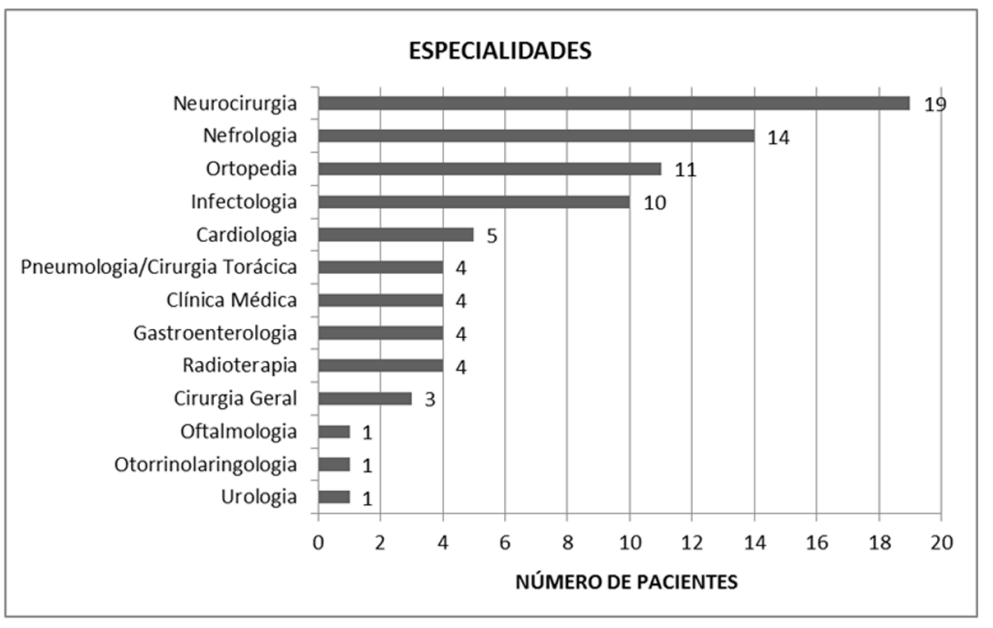

Os diagnósticos apresentados com maior frequência foram: osteomielites, Insuficiência Renal Crônica, Fraturas, Infecções de Sítio Cirúrgico, Traumatismo Cranioencefálico e Acidente Vascular Cerebral.
A Tabela 1 apresenta a distribuição das avaliações realizadas nas áreas do cuidado de acordo com a complexidade observada.

Tabela 1 - Distribuição das avaliações realizadas nas áreas do cuidado de acordo com a complexidade, Londrina-PR, 2010.

\begin{tabular}{|c|c|c|c|c|c|c|c|c|c|c|}
\hline \multirow[b]{3}{*}{ ÁREA DO CUIDADO } & \multicolumn{10}{|c|}{ GRAU DE COMPLEXIDADE } \\
\hline & \multicolumn{2}{|c|}{1} & \multicolumn{2}{|c|}{2} & \multicolumn{2}{|c|}{3} & \multicolumn{2}{|c|}{4} & \multicolumn{2}{|c|}{ Total* } \\
\hline & n & $\%$ & $\mathbf{n}$ & $\%$ & $\mathrm{n}$ & $\%$ & $\mathbf{n}$ & $\%$ & $\mathbf{n}$ & $\%$ \\
\hline Estado Mental & 758 & 81,9 & 119 & 12,8 & 12 & 1,3 & 37 & 4 & 926 & 100 \\
\hline Oxigenação & 615 & 66,4 & 167 & 18 & 119 & 12,9 & 25 & 2,7 & 926 & 100 \\
\hline Sinais Vitais & 3 & 0,3 & 909 & 98,2 & 6 & 0,6 & 8 & 0,9 & 926 & 100 \\
\hline Motilidade & 385 & 41,6 & 304 & 32,8 & 160 & 17,3 & 77 & 8,3 & 926 & 100 \\
\hline Deambulação & 239 & 25,8 & 264 & 28,5 & 100 & 10,8 & 323 & 34,9 & 926 & 100 \\
\hline Alimentação & 534 & 57,7 & 219 & 23,6 & 132 & 14,3 & 41 & 4,4 & 926 & 100 \\
\hline Cuidado Corporal & 258 & 27,8 & 325 & 35,1 & 10 & 1,1 & 333 & 36 & 926 & 100 \\
\hline Eliminações & 241 & 26 & 324 & 35 & 104 & 11,2 & 257 & 27,8 & 926 & 100 \\
\hline Terapêutica & - & - & 701 & 75,7 & 225 & 24,3 & - & - & 926 & 100 \\
\hline $\begin{array}{l}\text { Integridade da } \\
\text { pele }\end{array}$ & 256 & 27,6 & 168 & 18,2 & 415 & 44,8 & 87 & 9,4 & 926 & 100 \\
\hline Curativo & 283 & 30,6 & 107 & 11,5 & 485 & 52,4 & 51 & 5,5 & 926 & 100 \\
\hline Tempo de curativo & 283 & 30,6 & 184 & 19,8 & 289 & 31,2 & 170 & 18,4 & 926 & 100 \\
\hline
\end{tabular}

* Durante o período de coleta dos dados vários dos 81 pacientes foram avaliados até 11 vezes. 


\section{Classificação de pacientes}

Conforme observado na Tabela 1, em relação ao estado mental, $81,9 \%$ das 926 análises realizadas caracterizaram os pacientes como orientados no tempo e espaço; $66,4 \%$ constavam a não utilização de oxigênio em sua terapêutica, e 2,7\%, a utilização de ventilação mecânica.

Considerando o controle de sinais vitais, observou-se que $98,2 \%$ foram mensurados de 6 em 6 horas como 0 estabelecido pela instituição para pacientes que não necessitam de controles mais frequentes, e 1,5\% registraram a necessidade de verificações em intervalos menores que 4 horas, preconizado para pacientes graves.

Em relação à mobilidade dos pacientes, observou-se que $41,6 \%$ movimentavam todos os segmentos corporais, seguidos por $32,8 \%$ que apresentavam limitação de movimentos. Houve maior frequência de pacientes restritos ao leito $(34,9 \%)$, seguidos por pacientes que necessitaram de auxílio para deambular na realização de necessidades humanas básicas $(28,5 \%)$ (Tabela 1).

Da mesma forma, destaca-se o elevado número de atendimentos detectados enfatizando a necessidade da realização de banho no leito (36\%), seguidos de $35,1 \%$ que necessitaram de auxílio para o banho de chuveiro e/ou higiene oral. A elevada frequência de pacientes dependentes da equipe de enfermagem para sua locomoção e higiene corporal devese ao fato de muitos pacientes serem idosos, com sequelas neurológicas ou com fraturas, necessitando do auxílio da equipe de enfermagem para se locomoverem.

Em relação à terapêutica utilizada, $75,7 \%$ dos registros trazem a administração de medicamentos por via endovenosa intermitente, e 24,3\% em endovenosa contínua ou pela sonda nasogástrica. Não houve registro de pacientes que utilizavam apenas a via oral como forma de medicalização, nem a necessidade de administração de drogas vasoativas para manutenção da pressão arterial.

Conforme apresentado na Tabela 1, 0 item integridade da pele mostrou que $44,8 \%$ dos pacientes que apresentaram presença de solução de continuidade na pele, esta era de tecido subcutâneo e músculo, ou ainda incisão cirúrgica, ostomias ou drenos. Para recuperar a integridade da pele, $52,4 \%$ dos atendimentos mostraram necessidade de realização de curativo por duas vezes ao dia. A maior frequência para a realização dos curativos foi entre 15 e 30 minutos $(31,2 \%)$.

A Tabela 2 retrata a complexidade assistencial de acordo com as categorias de cuidado.

Tabela 2 - Categorias de cuidados de acordo com a graduação da complexidade assistencial da população estudada. Londrina-PR, 2010.

\begin{tabular}{lcc}
\hline CATEGORIA DE CUIDADO & $\boldsymbol{n}$ & \% \\
\hline Cuidado intensivo & 14 & 17,3 \\
Cuidado semi-intensivo & 18 & 22,2 \\
Cuidado de alta dependência & 24 & 29,6 \\
Cuidado intermediário & 13 & 16,0 \\
Cuidado mínimo & 12 & 14,8 \\
\hline TOTAL & $\mathbf{8 1}$ & $\mathbf{1 0 0 , 0}$ \\
\hline
\end{tabular}

Das avaliações realizadas, 29,6\% apresentaram correspondência com o intervalo das pontuações indicadas para a classificação dos pacientes na categoria do cuidado de alta dependência de enfermagem, seguida por cuidado semiintensivo $(22,2 \%)$ e intensivo (17,3\%).

\section{DISCUSSÃO}

0 hospital em estudo é referência no atendimento a pacientes que necessitam de atendimento de urgência e emergência. Os pacientes chegam a este serviço por intermédio do Serviço de Atendimento Móvel de Urgência (SAMU), Serviço Integrado de Atendimento ao Trauma e Emergências (SIATE), hospitais de nível secundário da região, Unidades Básicas de Saúde (UBS) ou por procura espontânea.

Desta forma, é possível ponderar que esta característica da instituição e o aumento progressivo de agravos por causas externas, com destaque para os acidentes de trânsito, podem estar relacionados com o elevado grau de dependência dos pacientes assistidos, muitos apresentaram diagnósticos relacionados a estes fatores.

Ressalta-se também que a unidade em estudo recebe pacientes que estão de alta da UTI. Para acompanhar a qualidade da assistência destes pacientes, faz-se necessário o uso de instrumentos como o de classificação dos pacientes.

Para os indicadores oxigenação e estado mental, verificou-se neste estudo que, respectivamente, $66,4 \%$ dos atendimentos apontaram para a não utilização de oxigênio e $81,9 \%$ indicaram orientação no tempo e espaço, sugerindo a literatura que o adequado nível de consciência e oxigenação implica melhor prognóstico, o que favorece o autocuidado. ${ }^{11}$

0 autocuidado dos pacientes do estudo é prejudicado pelo fato de $72,2 \%$ dos atendimentos indicarem necessidade de banho no leito ou auxílio para o banho no chuveiro, e 74,2\% estarem restritos ao leito ou necessitarem de auxílio para movimentar segmentos corporais ou deambular. Esses dados 
aproximam-se aos apresentados em estudo desenvolvido em UTI de um Hospital de Ensino do Paraná que trouxe em evidência que a maioria absoluta (quase a totalidade 99,62\%) dos pacientes recebe banho no leito por estar restrita a ele, seja pela patologia de base que exige limitação de esforços físicos ou pela incapacidade motora de locomoção ${ }^{12}$.

Em outro estudo, discute-se a questão dos pacientes ortopédicos que têm também uma notável e previsível dependência. Tais pacientes não dependem somente de cuidados físicos, mas também de apoio psicológico e emocional ${ }^{3}$.

Ainda em relação ao cuidado corporal, um estudo desenvolvido em Unidades de Internação e Unidades de Terapia Intensiva de um hospital de ensino no interior do estado de São Paulo demonstrou que este indicador crítico é o que apresenta maior peso fatorial, sendo o mais importante para a mudança na categoria do cuidado ${ }^{13}$, o que vem ao encontro dos achados do nosso estudo, pelos quais foram também os indicadores que contribuíram para a elevação do grau de dependência: motilidade, deambulação, cuidado corporal, eliminações, integridade da pele e curativo.

Para o indicador integridade cutânea, o estudo mostra que $80 \%$ dos pacientes apresentaram presença de solução de continuidade em uma ou mais áreas do corpo sem áreas de necrose ${ }^{12}$, resultado superior ao observado nesse estudo que identificou $44,8 \%$ de pacientes com presença de solução de continuidade da pele.

Em relação às categorias do cuidado, nosso estudo apontou maior frequência para o cuidado de alta dependência e semi-intensivo, díspar ao achado em estudo realizado no Hospital de Clínicas de Porto Alegre-RS, em unidade de internação, onde se identificou que a maioria dos pacientes pertencia à classificação de autocuidado e intermediários ${ }^{14}$.

Por se tratar de uma unidade internação, o perfil da clientela desse estudo deveria ser caracterizado por pacientes com cuidados mínimos e intermediários, o que não se verificou, pois $29,9 \%$ dos pacientes foram classificados como de alta dependência.

Após esta constatação, observou-se que muitos dos pacientes analisados apresentaram características de pacientes de UTI devido a suas condições de saúde, porém a falta de vaga nesta unidade especializada fez com que eles continuassem em unidade de internação. Esta constatação traz preocupações para os gestores, uma vez que cerca de $30 \%$ dos pacientes da unidade do estudo deveriam estar internados em UTI, setor hospitalar destinado ao atendimento de pacientes graves, com pessoal altamente capacitado e habilitado a oferecer o melhor tratamento e consequente recuperação ou cura do paciente, proporcionando um ambiente permeado por tecnologia de ponta e necessidade constante de agilidade e habilidade no atendimento ao cliente ${ }^{15}$, condições estas muitas vezes difíceis de serem proporcionadas em unidades de internação.

Neste estudo observou-se também uma assistência de enfermagem a desejar aos 17,3\% de pacientes com a categoria de cuidados intensivos; o dimensionamento do pessoal de enfermagem não se adequou a esta realidade.

Remetendo-se a situação da falta de leitos em UTI ao país, buscou-se saber como está a oferta deles em diferentes regiões, constatando-se que o Paraná tem 791 leitos de UTI Adulto que atendem pelo Sistema Único de Saúde (SUS) em 31 cidades, ou seja, apenas 7,7\% dos municípios dispõem do serviço, 367 municípios do estado não têm vagas em UTI para atender pacientes a partir de 15 anos em estado grave de saúde. Além da falta de leitos em algumas cidades e a concentração em outras, há também o problema da distribuição entre o serviço público (atendido pelo SUS) e o particular ${ }^{16}$. Esta falta de leitos em algumas cidades pode justificar a escassez de vagas em UTI nos hospitais da cidade onde 0 estudo foi desenvolvido, uma vez que esta atende pacientes referenciados de outras regiões.

0 que se observa é que esta política não está sendo efetivada de fato, com palpável fragilidade no sistema de saúde do Brasil, demonstrada pela falta representativa de leitos especializados que atendam às necessidades de cuidado da população.

\section{CONCLUSÃO}

0 presente estudo contribuiu para o conhecimento do grau de dependência dos pacientes de uma unidade de internação médico-cirúrgica e suas necessidades em relação aos cuidados de enfermagem.

Os resultados demonstraram que, apesar de 81,9\% dos atendimentos apresentarem pacientes orientados no tempo e espaço, 74,2\% estavam restritos ao leito ou necessitavam de auxílio para movimentar segmentos corporais ou deambular, com 72,2\% dos atendimentos indicando necessidade de banho no leito ou auxílio para o banho no chuveiro, e 44,8\% mostraram presença de solução de continuidade na pele envolvendo tecido subcutâneo e músculos, sendo realizados dois ou mais curativos ao dia com utilização de 15 a 30 minutos em sua realização. Evidenciouse o elevado grau de dependência dos pacientes internados, com amostra significativa de indivíduos com necessidade de cuidado de alta dependência e semi-intensivo.

0 estudo evidencia a necessidade de se repensar a estruturação da rede de saúde do SUS na região norte do Paraná, uma vez que a insuficiência de leitos de Unidades de Terapia Intensiva gera a necessidade de internamento de pacientes que exigem cuidados intensivos em unidades de internação, problema esse cada vez mais comum não só em hospitais de alta complexidade do município como também nos de média complexidade.

Essa situação leva a equipe de enfermagem a enfrentar dificuldades inerentes à mudança de grau de dependência dos pacientes destas unidades de internação exigindo um dimensionamento de pessoal diferenciado para atender à complexidade da assistência destes pacientes. 
São necessários estudos que aprofundem as dificuldades enfrentadas pela equipe de enfermagem ao prestar cuidados a pacientes graves em unidades de internação, além de se investigar as fragilidades que impedem a ampliação de leitos em unidades especializadas.

\section{REFERÊNCIAS}

1.Martins EAP, Haddad MCL. Validação de um instrumento que classifica os pacientes em quatro graus de dependência do cuidado de enfermagem. Rev Latino-Am Enfermagem. 2000 abr; 8(2): 74-82.

2.Fugulin FMT, Gaidzinski RR. Sistema de classificação de pacientes: análise das horas de assistência de enfermagem. Nursing, São Paulo, 1999 abr; 11(2): 27-34.

3.Alves MVMFF, Messora TCC, Gonçalves SPA, Luppi CHB. Avaliação do grau de dependência de pacientes em enfermaria de ortopedia de um hospital escola. Rev Eletr Enferm. [on-line]. 2011 out/dez; [citado 2012 jan 20]; 13(4): 612-19. Disponível em: http://www.revistas.ufg.br/ index.php/fen/article/view/9087/10182.

4.Tranquitelli AM, Padilha KG. Sistemas de classificação de pacientes como instrumentos de gestão em Unidades de Terapia Intensiva. RevEsc Enferm USP. [on-line]. 2007 [citado 2011 fev 03]: 41 (1): 141-46. Disponível em: http://www.ee.usp.br/reeusp/upload/pdf/314.pdf.

5.Antunes AV, Costa MN. Dimensionamento de pessoal de enfermagem em um hospital universitário. Rev Latino-Am Enfermagem. 2003 nov/dez; 11(6): 832-39.

6. Carmona LMP, Évora YDM. Grau de dependência do paciente em relação à enfermagem: análise de prontuários. Rev Latino-Am Enfermagem. 2003 jul/ago; 11(4): 468-73.

7.Laus AM, Anselmi ML. Caracterização dos pacientes internados nas unidades médicas e cirúrgicas do HCFMRP-USP, segundo grau de dependência em relação ao cuidado de enfermagem. Rev Latino-Am Enfermagem. 2004 jul/ago; 12(4): 643-49.

8.Souza GPS, Freitas GF, Prado C, Leite MMJ, Pereira IM. A problemática da elaboração da escala mensal de enfermagem. Acta Paul Enferm. 2011; 24(1): 137-41.

9. Rogenski KE, Fugulin FMT, Gaidzinski RR, et al. Tempo de assistência de enfermagem em instituição hospitalar de ensino. Rev Esc Enferm USP. 2011; 45(1): 223-29.

10.Santos F, Rogenski NMB, Baptista CMC, Fugulin FMT. Sistema de classificação de pacientes: proposta de complementação do instrumento de Fugulin, et al. Rev Latino-Am Enfermagem. 2007 set/out; 15(5): 10308.
11.Matsushita MS, Adami NP, Carmagnani MIS. Dimensionamento do pessoal de enfermagem das unidades de internação do Hospital São Paulo. Acta Paul Enferm. 2005; 18(1): 9-19.

12. Inoue KC, Matsuda LM. Dimensionamento da equipe de enfermagem da UTI:adulto de um hospital ensino. Rev Eletr Enferm. [on-line]. 2009; [citado 2011 mar 24]: 11(1): 55-63. Disponível em: http://www.fen.ufg.br/ revista/v11/n1/pdf/v11n1a07.pdf.

13.Perroca MG, Gaidzinski RR. Análise da validade de constructo do instrumento de classificação de pacientes proposto por Perroca. Rev Latino-Am Enfermagem. 2004 jan/fev; 12(1): 83-91.

14.Fonseca JP, Echer IC. Grau de dependência de pacientes em relação à assistência de enfermagem em uma unidade de internação clínica. Rev Latino-Am Enfermagem. 2003 dez; 24(3): 346-54.

15.Bolela F, Jerico MC. Unidades de terapia intensiva: considerações da literatura acerca das dificuldades e estratégias para sua humanização. Esc Anna Nery. [on-line]. 2006 [citado 2011 maio 19]; 10(2): 301-09. Disponível em: http://www.scielo.br/scielo.php?pid=S1414$81452006000200019 \&$ script=sci_arttext.

16.Milan P. UTIs mal distribuídas. Gazeta do Povo 2010 set 28. [citado 2011 abr 15] Disponível em: http://www.gazetadopovo.com.br/ vidaecidadania/conteudo.phtml?tl=1\&id=1049394\&tit=UTIs-maldistribuidas 